\section{Fixkombination für die Antiemese}

\begin{abstract}
In den Leitlinien zur Antiemese wird die gleichzeitige Gabe zielgerichteter prophylaktischer Medikamente empfohlen, die die am Erbrechen beteiligten Signalwege inhibieren. Eine dieser Medikationen ist die Fixkombination NEPA, deren Wirksamkeit in einer aktuellen Studie untersucht wurde.
\end{abstract}

Nom EPA ist eine orale Fixkombination aus dem neuen $\mathrm{NK}_{1}$-Rezeptorantagonisten Netupitant $(300 \mathrm{mg})$ und $\mathrm{Pa}-$ lonosetron $(0,50 \mathrm{mg})$, einem $5-\mathrm{HT}_{3}-\mathrm{Re}-$ zeptorantagonisten. In einem einzelnen Chemotherapiezyklus hatte sich NEPA in der Prävention von chemotherapieinduzierter Übelkeit und Erbrechen (CINV) oralem Palonosetron bereits als überlegen erwiesen. In der Phase-III-Extensionsstudie wurden nun Wirksamkeit und Sicherheit der Fixkombination über mehrere Zyklen untersucht.

In der doppelblinden Studie wurde eine Einzeldosis NEPA mit oralem $\mathrm{Pa}$ lonosetron bei zuvor chemotherapienaiven Patienten verglichen, die eine Chemotherapie mit einem Anthrazyklin und Cyclophosphamid zusammen mit
$12 \mathrm{mg}$ (NEPA-Gruppe) oder $20 \mathrm{mg}$ Dexamethason (Palonosetron-Gruppe) an Tag 1 erhielten. Primärer Endpunkt war das verzögerte vollständige Ansprechen (25-120 Stunden nach Gabe kein Erbrechen, keine Rescue-Medikation) in Zyklus 1. Die anhaltende Wirksamkeit wurde in der Extensionsphase über mehrere Zyklen untersucht, anhand des vollständigen Ansprechens (0-120 Stunden) in den Zyklen 2-4 und der Wahrscheinlichkeit eines anhaltenden vollständigen Ansprechens über mehrere Zyklen.

$88 \%$ der 1.455 randomisierten Patienten nahmen an der Extensionsphase über mehrere Zyklen teil. $76 \%$ erhielten $\geq 4$ Zyklen. Der Anteil an Patienten mit vollständigem Ansprechen war in den ersten
4 Zyklen unter NEPA im Vergleich zu Palonosetron signifikant höher (Zyklus 1: 74,3 vs. $66,6 \%$; Zyklus 2: 80,3 vs. $66,7 \%$; Zyklus 3: 83,8 vs. 70,3\%; Zyklus 4: 83,8 vs. $74,6 \%$; $\leq 0,001$ in jedem Zyklus). Der kumulative Anteil an Patienten mit anhaltendem vollständigem Ansprechen über alle 4 Zyklen war unter NEPA ebenfalls signifikant höher ( $\mathrm{p}<0,0001)$.

NEPA wurde auch über mehrere $\mathrm{Zy}$ klen gut vertragen. Die Gesamtinzidenz, Art, Häufigkeit und Intensität unerwünschter therapiebedingter Ereignisse war in beiden Gruppen vergleichbar.

Fazit: NEPA erwies sich als anwenderfreundliche antiemetische Fixkombination, die über mehrere Zyklen einer Chemotherapie wirksam und sicher ist.

Judith Neumaier

Aapro M et al. NEPA, a fixed oral combination of netupitant and palonosetron, improves control of chemotherapy-induced nausea and vomiting (CINV) over multiple cycles of chemotherapy: results of a randomized, double-blind, phase 3 trial versus oral palonosetron. Support Care Cancer. 2017;25(4):1127-35.

\title{
Krebsschmerzen: Oxycodon/Naloxon auch hochdosiert gut verträglich
}

\section{In einer Studie wurden Wirksamkeit und Verträglichkeit einer hochdosierten Therapie mit Oxycodon und Naloxon bei chronischen Krebsschmerzen unter- sucht.}

— ür eine erfolgreiche Schmerztherapie - mit Opioiden muss ihre analgetische Wirksamkeit die therapiebedingten $\mathrm{Ne}$ benwirkungen wie Benommenheit, Mundtrockenheit, Übelkeit und Obstipation überwiegen. Bis zu $90 \%$ der Patienten leiden unter einer Opioid-Therapie an Obstipation. Daher wird in den Leitlinien der Einsatz von Laxanzien empfohlen. Eine Alternative ist die orale Fixkombination des Opioidagonisten Oxycodon mit dem Opioidantagonisten Naloxon. In verschiedenen Studien zeigte diese Kombination eine vergleichbare Wirksamkeit wie Oxycodon allein, bei überlegener Verträglichkeit. Zur hochdosierten Therapie mit Oxycodon/Naloxon bei Patienten mit unkontrollierten
Schmerzen lagen bisher allerdings kaum Daten vor.

Diese liefert nun eine prospektive italienische Beobachtungsstudie, in der 119 Krebspatienten mit zuvor nicht kontrollierten moderaten bis schweren chronischen Schmerzen oder Unverträglichkeit gegenüber anderen Analgetika auf hochdosiertes Oxycodon/Naloxon ( $\geq 80 \mathrm{mg} / \mathrm{d}$ ) umgestellt wurden. Primärer Endpunkt war die Rate des analgetischen Ansprechens nach 30 Tagen (Abnahme der Schmerzintensität um $\geq 30 \%$ auf einer numerischen Ratingskala [NRS] von 0 bis 10). Die Ansprechrate lag nach 30 Tagen bei 79,8\%. Außerdem reduzierte die Therapie mit Oxycodon/Naloxon signifikant die Schmerzen nach 60 Tagen
(NRS T0: 7,4 vs. T60: 3,3; $\mathrm{p}<0,001$ ) und die Zahl der Tage mit Durchbruchschmerzen (3,9 vs. 2,0 Tage; $\mathrm{p}<0,001)$. Die tägliche Oxycodon/Naloxon-Dosis stieg leicht von $81,3 \mathrm{mg}$ zu Studienbeginn auf 93,6 mg an Tag 60 ( $p<0,001)$.

Der Einfluss der Schmerzen auf die Lebensqualität ließ im Studienverlauf signifikant nach $(\mathrm{p}<0,0001)$, und auch die Darmfunktion verbesserte sich mit der Zeit ( $\mathrm{p}<0,001)$. Nach Umstellung auf Oxycodon/Naloxon nahm die Zahl der Patienten mit Nebenwirkungen signifikant $\mathrm{ab}(\mathrm{p}<0,0001)$, ebenso die Anwendung von Laxanzien und Antiemetika.

Fazit: Oxycodon/Naloxon war auch in hohen Dosen bei Krebspatienten mit chronischem Schmerz hochwirksam und gut verträglich. Es linderte die Schmerzen schnell und reduzierte die OpioidNebenwirkungen.

Judith Neumaier

Amato $\mathrm{F}$ et al. High dosage of a fixed combination oxycodone/naloxone prolonged release: efficacy and tolerability in patients with chronic cancer pain. Support Care Cancer. 2017; https:// doi.org/10.1007/s00520-017-3709-5. 